\title{
USO Y VALORACIÓN DE LA TORTUGA \\ VERDE (CHELONIA MYDAS) EN LA COMUNIDAD DE AWASTARA
}

Maritza Cordón Suárez ${ }^{[1]}$

Enrique Cordón Suárez ${ }^{[2]}$

\section{Resumen}

Este estudio se enfocó en los aspectos socioculturales, económicos y legales que intervienen en el aprovechamiento de la tortuga verde (Chelonia mydas) en la comunidad indígena miskita de Awastara. Su metodología fue cualitativa y cuantitativa, en el paradigma de la investigación crítico social y el interaccionismo simbólico.

Según los registros, el 95 \% de la población de Awastara se dedica a la captura de tortugas durante todo el año. Esta práctica no sólo es para asegurar la alimentación comunal; sino también tiene un valor espiritual, es un legado de sus ancestros y a través de esto han preservado su cultura. La visión comunitaria actual no es regularizar la captura, sino integrar la participación comunitaria de forma equitativa.

Los sitios de pesca son los Cayos Miskitos, y es la ruta por donde viaja la tortuga desde Norte a Sur. Esta ruta es permanente y de obligatoria estadía para su alimentación y descanso. La captura comunal es de unas 1200 unidades mensuales y el consumo es de 80 y 100 unidades mensuales, el remanente es para abastecer los mercados de Bilwi. Los costos varían de 10,700 a 6,000 córdobas por jornada, con un promedio de 20-30 tortugas, lo cual proyecta una utilidad neta promedio de 17,500 córdobas, y por cada tortuga destazada 1,071 córdobas.

La mayoría de las leyes y normativas emitidas por el MARENA y otras instancias, están dirigidas a la protección de la tortuga, sin considerar la parte espiritual y cotidiana de la comunidad; no obstante, también existen medidas comunales de protección del recurso con la pesca artesanal.

Palabras claves: tortuga, uso, valoración, pesca artesanal, Cayos Miskitos, normativas.

\footnotetext{
${ }^{[1]}$ Docente de la Carrera de Administración de Empresas de la URACCAN. Máster en Desarrollo con Identidad. sumaco24@hotmail.com

${ }^{[2]}$ Investigador y Coordinador de Ingeniería Agroforestal de la URACCAN. Máster en Arte con Mención en Recursos Naturales. encordon@yahoo.com
} 


\section{Summary}

This study focused on the socio-cultural, economic and legal aspects that are involved in the use of the green turtle (Chelonia mydas) in the indigenous miskitu community of Awastara. The methodology was qualitative and quantitative, based on the paradigm of the social critic investigation and the symbolic interactionism.

According to the records, $95 \%$ of the population of Awastara is dedicated to the capture of turtles throughout the year. This practice is not only to ensure communal food, but it also has a spiritual value, is a legacy of their ancestors and through this they have preserved their culture. The current community vision is not to regularize the capture, but to integrate community participation in an equitable manner.

The fishing spots are the Miskito Keys (Cayos Miskitos) and this is the route where the turtle travels from North to South. This route is permanent and of mandatory stay for their food and rest. Communal capture is about 1200 units per month and consumption is 80 to 100 units per month, the remnant is to supply the markets of Bilwi. Costs vary from 10.700 to 6.000 cordobas per day, with an average of 20-30 turtles, which projected a net utility of 17.500 cordobas, and for each turtle butchered 1.071 cordobas. Most laws and regulations issued by MARENA and other instances are aimed at the protection of the turtle, without considering the spiritual part and daily life of the community; however, there are also communal measures to protect the resource with craft fishing.

Keywords: turtle, use, valuation, craft fishing, Miskito Keys, regulations.

\section{Introducción}

La tortuga verde (Chelonia mydas) por cientos de años es un recurso de gran importancia en los países de Centroamérica y el Caribe, principalmente por el valor de su carne. Algunos científicos calculan que cuando Cristóbal Colón llegó por primera vez a este mar habían cerca de 16 millones de tortugas verdes (Bjorndal et al., 1999), otros incluso creen que habían más de 660 millones (Jackson, 1997). Pero no sólo su carne es de gran valor, sino también desde el punto de vista bioecológico. Es una especie que tiene una distribución geográfica amplia, incorpora grandes cantidades de energía en forma de biomasa a los flujos de energía y ciclos de nutrientes en cualquier ecosistema que habita. Su valor biótico es de gran magnitud para el equilibrio de los ecosistemas.

Bjorndal (1997), menciona que estudios de captura y recaptura de tortugas verdes juveniles en la naturaleza indican que una tortuga verde dura entre 25 y 50 años para llegar a la madurez. Eso implica que muy pocos individuos pueden llegar a la madurez. El papel que juegan estos animales en las economías locales y en varios casos 
regionales está supeditado a los bienes y servicios que ellas brindan a los pobladores de las costas; su explotación desde hace siglos como fuentes de carne, huevos, materia prima para cosméticos, joyería y artesanía y, en las últimas décadas, como elementos de atracción para el turismo.

A pesar de las magníficas cualidades de estas especies, se encuentran perjudicadas por diversos factores que han declinado sus poblaciones, entre ellos esta: la sobrepesca comercial, la captura incidental, la destrucción de hábitats críticos de alimentación, de anidación y de reposo y, más recientemente, la contaminación de los mares, todos han diezmado la condición actual de las tortugas marinas. Es así que esta tortuga ha sido enlistada como especie en severo peligro de extinción por el Libro rojo de laUnión Internacional para la Conservación de la Naturaleza(UICN) y en el apéndice I de Convención sobre el Comercio Internacional de Especies Amenazadas de Fauna y Flora Silvestres(CITES; citado por MARENA, 2005).

En la Región Autónoma Atlántico Norte (RAAN), la captura de tortugas es una actividad de gran relevancia sociocultural y económica. La carne es apetecida por los pobladores de los centros urbanos, principalmente Bilwi y Waspam. En cambio en las comunidades de los litorales se utiliza como alimento familiar y es una actividad de gran valor comercial al abastecer a los centros urbanos de carne.

La comunidad indígena miskita de Awastara es de mención especial, debido a que es considerada la comunidad representativa en esta actividad. Existe un involucramiento total de las familias (el $95 \%$ de la población comunal), presenta el mayor número de unidades de pesca por temporada, es la principal abastecedora de los mercados de Bilwi (MARENA; 2008), y se reporta una captura de más de 600 individuos por temporada de pesca autorizada en Awastara, y se supone que puede ser el doble la cantidad capturada sin autorización institucional. Se cree que la presente población de tortugas marinas es insuficiente para la sustentabilidad económica, biológica, ecológica, cultural o espiritual de la comunidad.

Estas características de la comunidad relacionada a la captura de la tortuga verde, ayudaron a enfocar este proyecto de investigación que se dirige al estudio de la relación sociocultural, económica y legal de los comunitarios miskitos de Awastara. En la realización de este estudio se analizan las interacciones entre los aspectos biológicos, sociales y económicos que influyen en la utilización de la tortuga, para luego conocer la posibilidad de hacer un uso racional, con base a los medios y capacidades existentes localmente. 


\section{Objetivos}

\section{General:}

Conocer los aspectos socioculturales, económicos y legales que intervienen en el aprovechamiento local de la tortuga verde (Chelonia mydas) en la comunidad indígena miskita de Awastara.

\section{Específicos:}

1. Describir los procesos de organización, captura y uso tradicional de la tortuga que los pescadores practican.

2. Analizar el proceso de comercialización y beneficios locales de los pescadores.

3. Analizar la aplicación del marco legal vigente relacionado a la protección, conservación y captura de la tortuga, sobre la base de la percepción comunitaria.

4. Identificar algunas medidas comunales de conservación y aprovechamiento de la tortuga.

\section{Revisión de literatura}

\section{Descripción de la especie}

Según Chacón (2001), en todo el mundo hay siete especies de tortugas marinas, cinco de las cuales están presentes en Nicaragua. La tortuga verde (Chelonia mydas), toma su nombre del color de su grasa, es la más grande de las tortugas de caparazón duro. También es la tortuga marina de más lento crecimiento, con un estimado de 27 a 50 años el llegar a la madurez sexual (Limpus \& Walter, 1980; Balazs, 1982; Frazer y Ehrhart, 1985; Frazer \& Ladner, 1986). Los adultos llegan a pesar de 135 a16o kg, con registros históricos de pesos de hasta $385 \mathrm{~kg}$ (Rebel, 1974). En el caso de la comunidad de Awastara, los registros mencionan individuos con pesos variados de 60-120 kg (MARENA, Informe anual 2009).

\section{La pesca artesanal de tortuga}

La pesca artesanal representa una actividad que aporta beneficios alimenticios y económicos a la familia, y que se caracteriza por mantener estrechos vínculos entre el pescador y su ambiente. La experiencia de la comunidad de Awastara, refleja que los pescadores tienen un amplio conocimiento empírico sobre el comportamiento de los recursos. 


\section{Valor y uso de la tortuga}

En el caso de la comunidad de Awastara, hablar sobre la valoración de la vida silvestre es hablar sobre las manifestaciones del uso y beneficios que aportan a los grupos que lo utilizan. Pérez - Gill (1996), considera que el uso de la fauna silvestre está representado por todas aquellas actividades a través de las cuales los seres humanos obtienen beneficios, ganancia, fruto o disfrute de una población biológica, sea de modo directo, indirecto, consuntivo o no consuntivo.

\section{Materiales y métodos}

\section{Apertura de la información}

La idea inicial de esta investigación surgió debido a que soy funcionaria del Ministerio de Ambiente y Recursos Naturales (MARENA), y de manera constante observaba a pescadores y destazadores presentarse a esta oficina a solicitar permisos de pesca y destace. Logré visualizar que existe abundante información en la memoria de los comunitarios, por lo que considere necesario iniciar un proceso de recopilación, organización y escritura de la misma.

Trasmití la idea del estudio a las autoridades de la URACCAN y a los comunitarios de Awastara, para la aceptación del tema. En este proceso también presenté la importancia social y ambiental que representaba el desarrollo de esta investigación, lo cual me permitió lograr la aprobación de ambos sectores para su realización.

\section{Universo de estudio y muestra}

El universo de estudio corresponde a todos los pescadores y pescadoras de la comunidad, que son en total 438 personas.

La muestra fue de 35 personas, distribuidas de la siguiente manera: 22 pescadores varones, 5 pescadoras mujeres (aunque su participación está enfocada en la comercialización de la tortuga (viva o destazada) y la pesca de camarón, 3 líderes comunales (Wihta, Síndico y un Consejo de Ancianos), que también son pescadores y 5 representantes de instituciones regionales (SERENA, ALCALDÍA, MARENA, FUERZA NAVAL, INPESCA). 


\section{Metodología}

El estudio abarcó un período de seis meses de trabajo de campo (mayo a octubre), con visitas periódicas a la comunidad, a los destazadores y a las instituciones públicas que tienen relación directa con el tema.

En un primer viaje a la comunidad de Awastara se logró realizar un grupo focal de 10 comunitarios y comunitarias para la presentación del tema de investigación y las consideraciones comunales, tomando en cuenta el aspecto intercultural de la investigación.

En el estudio se abordó la pesca artesanal, percepción comunitaria y valoración de la actividad de captura de la tortuga, de las cuales se obtuvo información cualitativa y cuantitativa sobre las tortugas, pescadores y usuarios. Se utilizaron las técnicas de: Observación participante (comunidad y destazadores), entrevistas y grupo focal.

Para darle salida al primer objetivo se desarrollaron las siguientes actividades. Se realizó un segundo viaje a la comunidad de Awastara en donde fueron entrevistados 22 pescadores, para conocer su proceso de organización en la jornada de trabajo y método de captura empleado. Asimismo, con el fin de conocer su participación en el proceso de acompañamiento en la jornada de trabajo o bien de comercialización.

Las actividades metodológicas que guiaron el segundo objetivo fueron ocho entrevistas a ocho pescadores de la comunidad, para conocer el proceso de comercialización de la tortuga viva y al destace. Y, en Bilwi, se entrevistó a cuatro destazadores y comerciantes de carne fresca de tortuga, para conocer los precios de costo y valorar el rendimiento al destace.

En Bilwi hice presencia durante el destace de tres tortugas hembras en el barrio El Muelle (03:00 am), con el fin de hacer el pesaje de las partes de cada una de ellas. Esto me permitió determinar el peso promedio por tallas, parámetro necesario para desarrollar una valoración del análisis financiero. A partir de esto, se hizo un análisis económico del proceso de comercialización, y los ingresos obtenidos por los comunitarios y destazadores.

Para darle salida al tercer objetivo se recopilaron normativas nacionales y regionales que están vigentes: Ley de Pesca de 1964, Decreto de 1958 (aún vigente), Decreto Ministerial 043-2005 del MARENA, Ley No. 445, Ley No. 28, disposición administrativa del gobierno regional No. 69-23-01-2006, entre otras. De igual manera se entrevistaron a funcionarios institucionales que tienen vínculo con la aplicación de las leyes: MARENA, SERENA, NAVAL, ALCALDIA MUNICIPAL y PGR. Cada uno de ellos informó sobre sus funciones y competencias relacionadas a la regulación del recurso en estudio. 


\section{Resultados y discusión}

La comunidad de Awastara pertenece al territorio Tawira en el litoral Norte, municipio de Puerto Cabezas y se encuentra ubicada a $83^{\circ} 41.30^{\prime}$ longitud Oeste y a $14^{\circ} 40.16^{\prime}$ latitud Norte y a una distancia lineal de $61 \mathrm{Km}$ de la cabecera municipal de Bilwi. Esta comunidad lleva el nombre de Awastara porque en época pasada existía un enorme árbol de pino en el centro de la misma. La palabra Awastara es en lengua miskitu, y cuyo significado en español es Awas (Pino) y Tara (Grande) (Diagnóstico de Tawira, 2009: 18).

\section{La tradición pesquera de la tortuga}

Al indagar sobre la tradición de la actividad de captura de la tortuga, los comunitarios mencionan que es una actividad que se practica desde épocas ancestrales y que ahora forma parte de su vida diaria. Un comunitario de 54 años nos explicaba:

La pesca de tortuga se ha venido practicando desde antes de que vinieran los españoles, nosotros ya usábamos la tortuga (comunitarios), para esto no se necesita una práctica o un don especial, esto se aprende y se transmite a través del habla y la práctica en el mar por los padres o abuelos que también nos enseñaron como pescar desde muy pequeños. Aprendimos que si no se pesca no se come...

Un miembro del Consejo de Ancianos de la comunidad de Awastara menciona sobre los materiales, equipos y forma de pescar de sus ancestros, los cuales utilizaban materiales del entorno local que les proveía la naturaleza y que existía en la comunidad, combinado con el conocimiento tradicional del arte de pescar.

Los viejos de antes usaban Sani (corteza del árbol capulin: Trichospermum mexicani) y Pohlak dusa (árbol flotador de nombre común: balsa, guano, tambor: Ochroma pyramidable) para hacer las redes y flotadores, ahora con los proyectos que han venido es que usamos redes, boyas y veleros... No habían veleros, solamente habían unos botes de madera y canalete que no corrían mucho, entonces ellos se ponían en la punta de estos barcos y lanzaban un arpón o lanza a las aletas de las tortugas,... la pesca era sólo para alimento de la comunidad, en esos tiempos nadie pensaba en Bilwi, todo se quedaba en la comunidad, era para comer la carne con la familia.

\section{La organización comunitaria y la captura de tortugas}

Los resultados reflejan que el $95 \%$ de las familias se dedican a la actividad de captura de las tortugas durante todo el año y un $5 \%$ a las actividades de comercialización (pulperías). También se observó que no existe una estructura comunal para la regulación 
de la captura de las tortugas. El síndico regula el uso de los recursos naturales, y por ende, su función se ha concentrado en regular la salida de las embarcaciones.

La norma comunal indica que todo miembro de la comunidad tiene permitido la captura de tortuga libremente durante todo el año. Sin embargo, se debe respetar el período de veda establecido por las instituciones del Gobierno Regional.

\section{Épocas y sitios de pesca}

Muchas teorías se han sugerido para explicar la habilidad de migración de las tortugas marinas por miles de kilómetros en los Océanos, desde sus áreas de alimentación hasta sus áreas de reproducción y anidación. Esta migración define la presencia de individuos en determinados períodos del año.

Carr, Carr \& Meylan (1990:49), anotan que la colonia de tortugas verdes que anidan masivamente en playa Tortuguero, migran de dos frentes; el Norte donde está el banco miskito de Nicaragua y del Sur, de las costas bajas de Venezuela y las Antillas menores; el 70.5 por ciento de las tortugas de Nicaragua arriban en julio, mientras que el 63.9 por ciento de los emigrantes del Sur del Caribe arriban en el mismo mes.

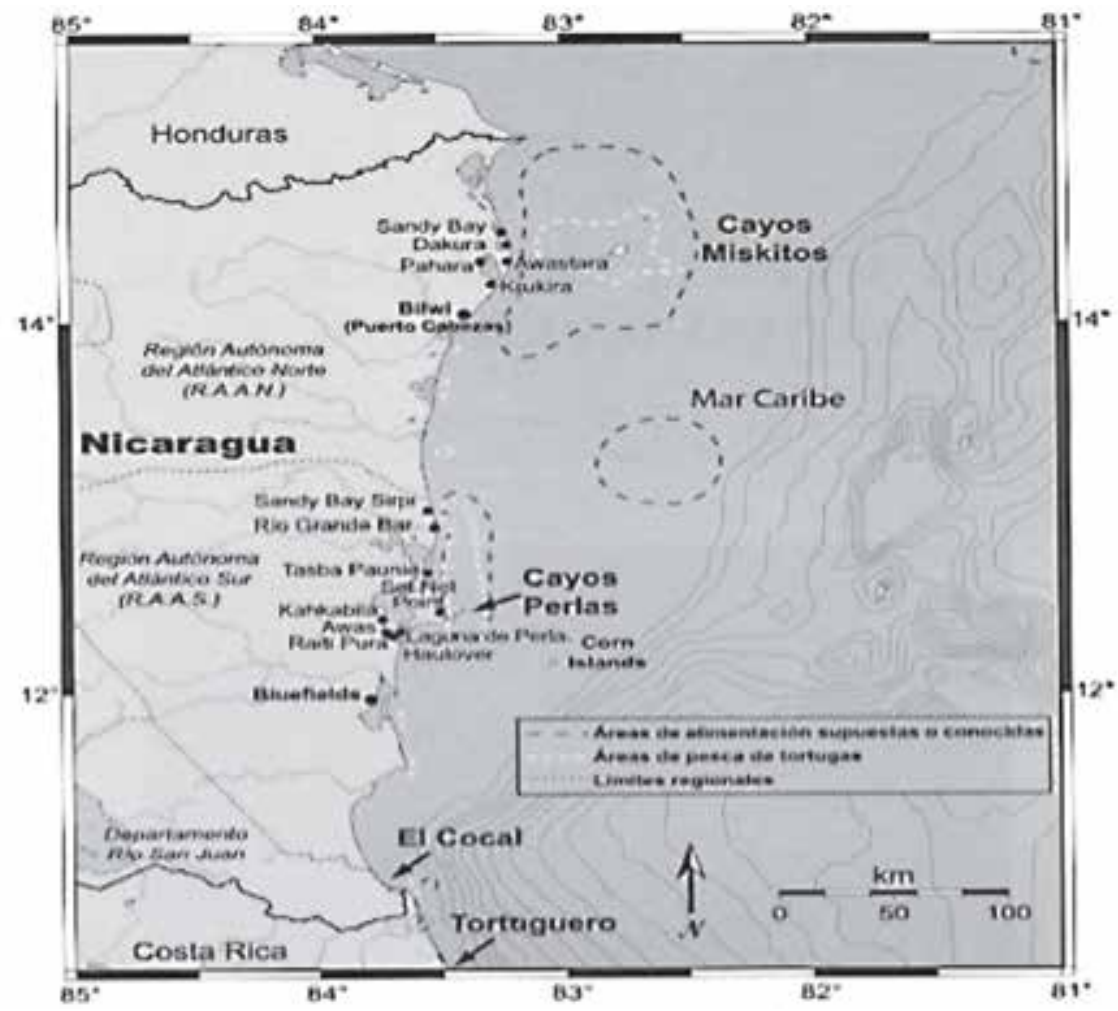

Mapa No. 1 Sitios de captura de la tortuga Verde, fuente: Lautex 
En relación a los sitios de pesca, estos se concentran en los alrededores de los Cayos Miskitos, $27 \mathrm{Km}^{2}$ situados mar adentro de la costa nordeste caribeña, en donde la ruta de viaje de la tortuga se orienta de Norte a Sur, a través de un corredor de viaje.

Los pescadores mencionaron cinco sitios principales de captura que cubre un radio de $15 \mathrm{~km}^{2}$, y son los siguientes: Limarka, Witis, Saudisrat, Papta y Racifales. Estos sitios tienen poca profundidad y abundantes rocas cubiertas de vegetación herbácea acuática (algas) y sirve de alimento a las tortugas. Ellas salen a alimentarse entre las 3:00 pm., durante este proceso natural es que se da la captura.

\section{Ruta migratoria}

Cruz, Galeano \& Espinal (1989), hacen una explicación clara de la ruta de las tortugas verdes que anidan en Costa Rica. Entre julio y octubre anidan en el Tortuguero. Durante una temporada de anidación, cada hembra deposita entre uno y seis nidos con intervalos de 10 a 14 días. Esta especie es común observarla en aguas alrededor de las Islas de Honduras, así como en los Cayos frente a la costa de la Mosquitia de Honduras donde también se encuentran los Cayos Miskitos de Nicaragua, sitio que los comunitarios de Awastara mencionan como sitios de captura tradicional de tortugas.

Al consultar con los comunitarios sobre la presencia de las tortugas, se menciona lo siguiente: "siempre hay tortugas... todo tamaño, grandes y chiquitos... los más grandes siempre están los meses de marzo a octubre”.

\section{Artes de pesca y captura}

El uso de los equipos está en dependencia de los métodos de captura empleados. No obstante, los materiales básicos más usados son: el velero de madera, redes, boyas, rocas y cuerdas.Uno de los miembros del Consejo de Ancianos nos explica sobre el proceso de organización previo a la jornada de pesca:

El grupo de pesca consta generalmente de cuatro tripulantes..., un capitán, un cocinero y dos ayudantes de pesca... se prepara para una faena de pesca de 10 días en el mar... se carga de provisión, leña, agua, todos los víveres que se necesita para trabajar en el mar porque ya saliendo mar adentro, ya no hay donde conseguir otra cuestión.

Se observa que el equipo conformado para la pesca es mínimo, así como los equipos de pesca son los típicos usados en la pesca artesanal. 


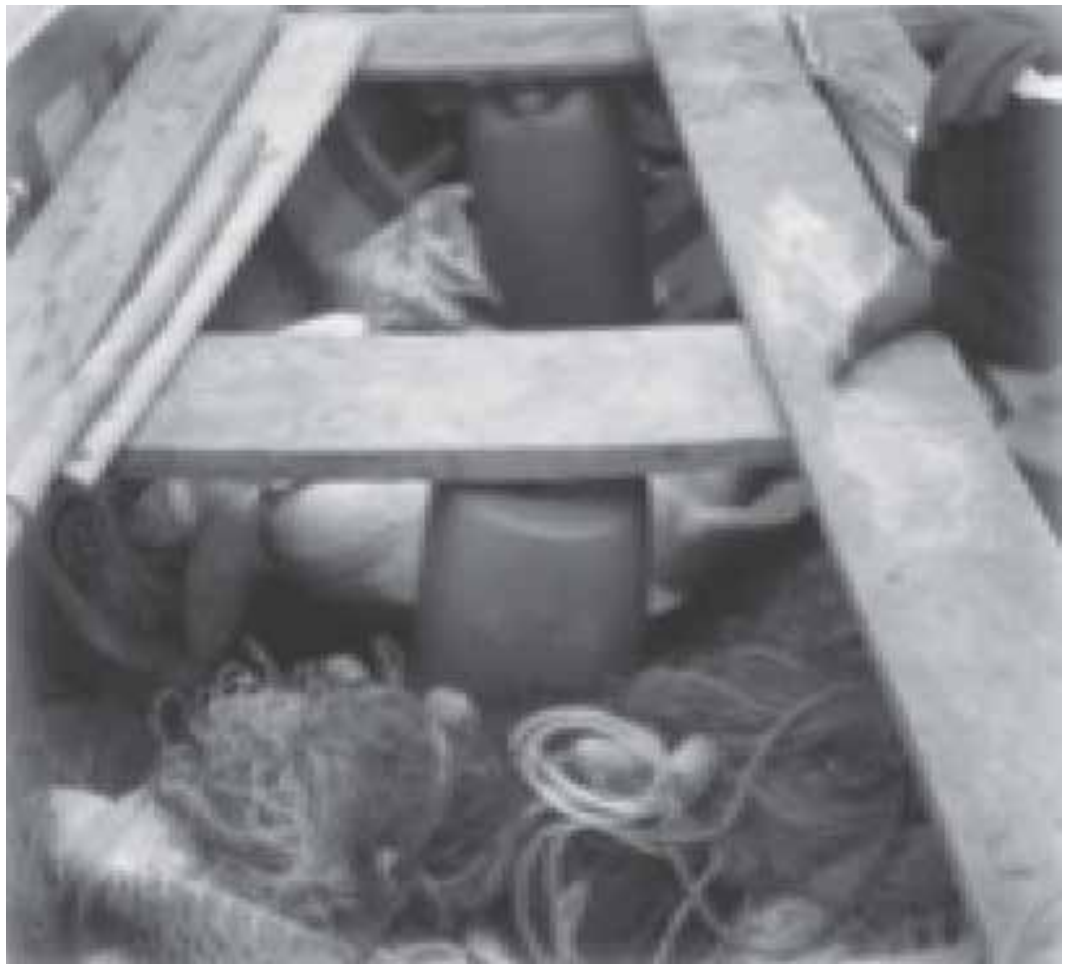

Foto No. 1 Instrumentos de pesca utilizadas por los comunitarios de Awastara. Tomada por Maritza Cordón 22/09/10.

El proceso de captura lo describe un comunitario:

... Las tortugas generalmente duermen en las piedras, ellas se sumergen al agua por una hora aproximadamente y luego salen a respirar, no diario se encuentran las tortugas o sea es cuestión de suerte... se enganchan piedras, que en miskitos se les llama kialingo, son unos pedazos de piedra que van amarradas con un mecate y una boya; entonces, esas boyas tienen un gancho donde se enganchan esas redes, cuando las tortugas salen a respirar se enredan en esas redes, a las 5:00 am vamos a revisar y vemos algunas enredadas en las redes, entonces se jalan con un garfio y se montan en el velero.

\section{El autoconsumo y otros usos}

En relación a los usos que los comunitarios le dan a los productos de la tortuga, se mencionan básicamente dos tipos: El de consumo como alimento y el de comercialización de la carne o sea la tortuga viva. En cuanto al consumo promedio por familia va en dependencia del número de miembros por familia. 


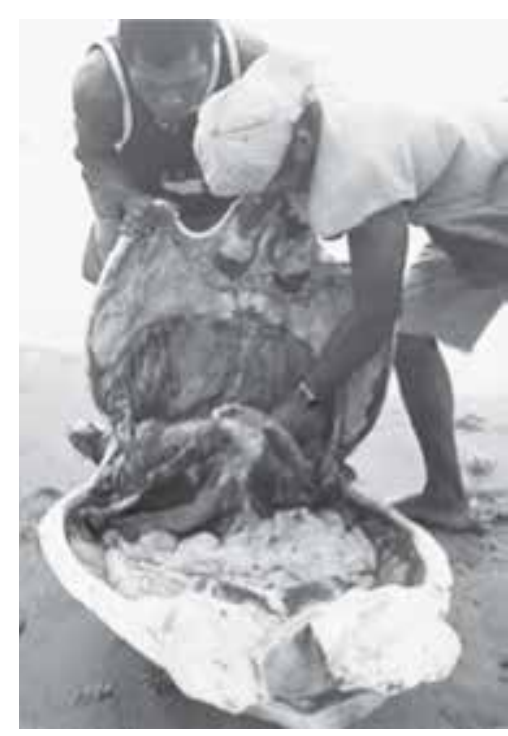

Foto No. 4 Destace de la Tortuga.

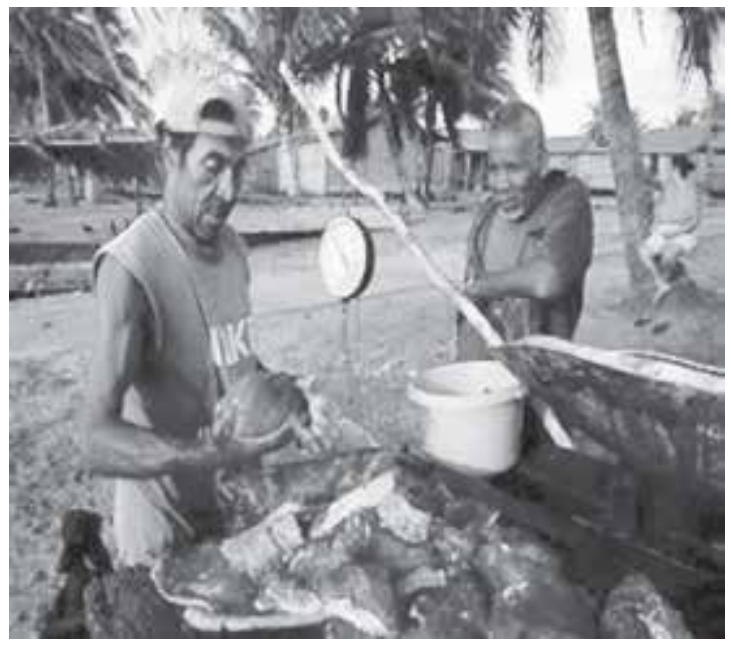

Foto No. 5 Comercio de carne en el Bo. El Muelle.

Comunidad de Awastara, foto tomada por Howard Enríquez, 20/09/10.

\section{Valor espiritual}

La leyenda de los indígenas miskitus cuenta que el tímido espíritu de la Madre Tortuga fue molestado por el hombre, y ella ascendió la montaña y se escondió en una cueva profunda y oscura. Algunos pescadores mencionan que la Madre Tortuga migró al Tortuguero, Costa Rica, la colonia principal para la tortuga verde que forrajea en Nicaragua. También se creía que ella podía afectar los movimientos de las tortugas y la suerte de los pescadores.

Fueron muy interesantes las opiniones de las personas de la comunidad al respecto. La mayoría expuso que sí tienen algunas creencias que se relacionan principalmente con la pesca, el destace y el consumo. Una comunitaria, tiene algunas creencias que surge del destace: "si una tortuga llora al matarla, seguramente su carne no tiene el mismo sabor, también si la destazamos y encontramos una pequeña piedra blanca, esto es señal de que no tendré suerte en la caza por un larga temporada".

También existe otra creencia: “... si tengo a mi esposa embarazada no puedo ir a cazar, porque siempre las tortugas se corren y no las pueden pescar”.

Pero también explican que se tiene la solución a estos problemas. Por ejemplo: ... Cuando andan sin poder cazar, los hombres se amarran unos pedazos de mecate en los pies y en las manos para ahuyentar la mala suerte..., después de eso empiezan a cazar normalmente la tortuga, ese es el secreto que tienen las tortugas. 
No se deja de mencionar y reconocer que el consumo de carne de tortuga tiene propiedades afrodisíacas. Los comunitarios lo relacionan con la relación de pareja y dicen:

Entre los animales, las tortugas son las que duran más para reproducirse, por eso las personas que comen abundante carne les afecta la sangre, a veces son infieles, este efecto es porque la tortuga es resistente cuando está con su pareja, duran encima de la tortuga hembra entre 1-2 meses, y el macho se baja cuando está débil, no puede respirar. Si usted agarra esa tortuga no sirve para comer porque no tiene carne, está seca y blanca, tiene un sabor aguado. Por eso también cuando un hombre o mujer está débil agarramos la cola de la tortuga hembra y le damos de comer tres o cuatro veces, para que se reponga.

Es importante destacar que las creencias han cambiado con el tiempo, en el pasado las creencias eran más mitológicas y tenía su principio en la conservación de la tortuga y en su población como elemento importante en la naturaleza y en la vida de los comunitarios.

\section{La comercialización}

La mayoría de los pescadores hicieron referencia a la comercializan de la tortuga de dos maneras. La primera forma es directamente en la comunidad. Esta es destazada y vendida por libras a 15.00 córdobas por libra (o.70 dólares). El segundo caso es la comercialización viva de la tortuga en la comunidad o en el muelle de Bilwi. Los costos varían de 1,200.00 - 2,000.00 córdobas (60-100 dólares).

\section{Rendimiento de pesca}

El rendimiento de pesca es una variable que presenta la relación de costos en que incurre el comunitario durante una jornada de pesca de 10 días promedio y la producción o número de ejemplares capturados, como se refleja a continuación:

Tabla No. 1: Gastos promedios realizados por jornada de pesca de 10 días.

\begin{tabular}{|l|c|c|c|}
\hline \multirow{2}{*}{\multicolumn{1}{|c|}{ Rubro }} & \multicolumn{3}{c|}{ Gastos C\$ } \\
\cline { 2 - 4 } & Pescador $\mathbf{1}$ & Pescador 2 & Pescador 3 \\
\hline Alimento & $1,200.00$ & 800.00 & 800.00 \\
\hline Alquiler de bote (préstamo de bote) & $1,000.00$ & 800.00 & 700.00 \\
\hline Alquiler de aperos de pesca & 500.00 & 500.00 & 500.00 \\
\hline Mano de obra (2 marinos, un cocinero y capitán) & $8,000.00$ & $5,500.00$ & $4,000.00$ \\
\hline \multicolumn{1}{|c|}{ TOTAL } & $\mathbf{1 0 , 7 0 0 . 0 0}$ & $\mathbf{7 , 6 0 0 . 0 0}$ & $\mathbf{6 , 0 0 0 . 0 0}$ \\
\hline Producción promedio (no. tortugas capturadas) & $15-20$ & $15-20$ & $10-15$ \\
\hline
\end{tabular}

Fuente: Datos recopilados de entrevistas a comunitarios pescadores 


\section{RECURSOS NATURALES Y MEDIO AMBIENTE}

Tabla No. 2: Análisis financiero, por jornada de pesca.

\begin{tabular}{|l|c|c|c|c|}
\hline \multicolumn{1}{|c|}{ Acción } & Pescador 1 & Pescador 2 & Pescador 3 & Promedio \\
\hline $\begin{array}{l}\text { Producción promedio } \\
\text { (no. Tortugas) }\end{array}$ & 18 & 15 & 12 & 15 \\
\hline Costo de venta & $2,000.00$ & $2,000.00$ & $2,000.00$ & $2,000.00$ \\
\hline $\begin{array}{l}\text { Utilidad bruta (Ingreso } \\
\text { total) }\end{array}$ & $36,000.00$ & $30,000.00$ & $24,000.00$ & $30,000.00$ \\
\hline Gastos operativos & $16,500.00$ & $12,000.00$ & $9,000.00$ & $12,500.00$ \\
\hline \multicolumn{1}{|c|}{ Utilidad neta } & $\mathbf{2 0 , 0 0 0 . 0 0}$ & $\mathbf{1 8 , 0 0 0 . 0 0}$ & $\mathbf{1 5 , 0 0 0 . 0 0}$ & $\mathbf{1 7 , 5 0 0 . 0 0}$ \\
\hline
\end{tabular}

Fuente: Datos recopilados de entrevistas a comunitarios pescadores

Tabla No. 3: Rendimiento de tres Tortugas verde (destace).

\begin{tabular}{|c|c|c|c|c|}
\hline Rubro & Ejemplar No. 1 (Lb) & Ejemplar No. 2 (Lb) & Ejemplar No. 3 (Lb) & Promedio \\
\hline Cabeza & 10.5 & 11.5 & 12.5 & \\
\hline Cola & 7.0 & 8.5 & 9.5 & \\
\hline Carne & 50.0 & 56.5 & 60.0 & \\
\hline Aleta & 5.0 & 6.5 & 8.0 & \\
\hline Riñones & 1.0 & 1.5 & 1.0 & \\
\hline Hígado & 3.0 & 3.5 & 5.0 & \\
\hline Pulmón & 2.0 & 2.5 & 3.5 & \\
\hline Corazón & 1.0 & 1.5 & 2.5 & \\
\hline Calipee & 3.0 & 4.5 & 5.5 & \\
\hline Caparazón & 12.5 & 14.5 & 16.5 & \\
\hline Intestinos & 10.0 & 11.0 & 13.5 & \\
\hline TOTAL & 106.5 & 121.0 & 137.0 & 121.5 \\
\hline
\end{tabular}

Fuente: Datos recopilados de entrevistas a comerciantes de Bilwi.

Tabla No. 4: Valoración del análisis financiero por destace de tortuga.

\begin{tabular}{|c|c|c|c|c|}
\hline Acción & Tortuga No. 1 & Tortuga No. 2 & Tortuga No. 3 & Promedio \\
\hline Libras al destace & 106.5 & 121 & 137 & 121.5 \\
\hline Costo de venta & 25.00 & 25.00 & 25.00 & 25.00 \\
\hline Valor de la venta & $2,665.50$ & $3,025.00$ & $3,425.00$ & $3,038.00$ \\
\hline Precio de compra & $1,600.00$ & $1,800.00$ & $2,500.00$ & $1,966.60$ \\
\hline Utilidad neta & $\mathbf{1 , 0 6 5 . 5}$ & $\mathbf{1 , 2 2 5 . 0 0}$ & $\mathbf{9 2 5 . 0 0}$ & $\mathbf{1 , 0 7 1 . 4}$ \\
\hline
\end{tabular}

Fuente: Datos recopilados de entrevistas a comerciantes y alcaldía

Estas tablas son un detalle de los gastos y beneficios que genera la tortuga por faena de pesca. 


\section{Marco legal y normativas de conservación de la tortuga}

En Nicaragua, desde 1958, vía decreto, se reglamentó el aprovechamiento de los huevos de tortugas, y se prohibió la destrucción de las tortugas. Por la Ley de Pesca de 1964, la tortuga se consideró recurso comercial a gran escala. Con la ratificación de CITES, restringió la exportación de este recurso. El decreto que se halla vigente, es el de 1958, que el Ministerio de Recurso Naturales y Ambiente (MARENA)implementa. Así mismo por los acuerdos administrativos de la autoridad nacional ambiental de 1980 y 1982, se norma acerca de los períodos de veda. Pero, además de los sistemas de vedas, en el 2006 el MARENA extiende un decreto ministerial número 043 - 2005 donde establece veda indefinida de las Tortugas Marina por su sobrexplotación en los últimos años. No obstante, el Gobierno Regional emite una Disposición Administrativa No. 69 23- 01 - 2006 contraria al Decreto Ministerial, debido a que se está violentando los derechos de los Gobiernos Regionales y de las comunidades indígenas.

Durante el desarrollo del grupo focal comunal, estos coincidieron en lo siguiente:

Con eso de la veda a nosotros ni nos avisaron que lo iban a hacer, solo cuando llegamos a vender las tortugas en Bilwi, el MARENA y la Alcaldía nos dijo que van a poner un tiempo en el que las tortugas no se pueden vender y que no podíamos llevarlas a vender o nos la iban a quitar...

Uno de los miembros del Consejo de Ancianos de Awastara dice:

Cuando hicieron eso de la veda no nos llamaron a reunión de consulta si queríamos esa veda o no, y a nosotros de nada nos sirve que haya veda porque nosotros siempre cazamos tortugas para nosotros y para nuestros hermanos comunitarios, desde que existe Bilwi únicamente problemas nos traen con sus leyes que nada resuelven...

Esa es la razón por la que en la comunidad de Awastara no toman en cuenta la ley de veda, sino las leyes comunales que ellos establecen de acuerdo a su cultura y creencias.

\section{Conclusiones}

Los aspectos socioculturales, económicos y legales que intervienen en el aprovechamiento local de la tortuga verde (Chelonia mydas) en la comunidad de Awastara, tienen su base en que es una actividad que se viene practicando desde épocas ancestrales y ahora forma parte de su sistema de vida tradicional. Está fuertemente relacionado al vínculo familiar, con sus creencias y con la respuesta a una necesidad básica de alimentarse. 
La estructura comunal tradicional se enfoca en regular la captura de tortugas, autorizando el método de pesca artesanal y permitiendo la participación de todos los comunitarios.

La pesca de tortuga actualmente es una actividad con alto valor comercial y con la cual los comunitarios crearon dependencia económica. Actualmente existe un mercado establecido y la pesca está sujeta a ciertas normas de regulación local e institucional. Su comercialización se hace al destace en la comunidad y en Bilwi en forma viva y al destace.

No se lleva un registro formal del número de capturas en la comunidad, ni del consumo, pero según los comunitarios la captura anda por las 20-30 tortugas por faena de pesca. En el caso del consumo comunal anda por las 80 y 100 unidades mensuales, lo cual equivale al destace de 3-4 tortugas diarias. De igual manera existe una utilidad neta promedio por jornada de pesca de 17,500.00 córdobas y por cada ejemplar destazado un promedio de 1,071.00 córdobas.

La mayoría de las leyes y normativas están dirigidas hacia la protección de la tortuga, sobre la base de la regulación del uso y no considera las formas de vida cotidiana y tradicional de la comunidad que depende del dinamismo de la tortuga.

\section{Lista de referencia}

Asamblea Nacional de la República de Nicaragua. Ley de pesca 489 y su reglamento. La Gaceta No. 251 del 27 de Diciembre del 2004.

Balazs, José (2004). Tipos de tortugas de la Región Centro Americana. $200-203$.

Bjorndal \& Carr (1989). Técnicas de Investigación y Manejo para la Conservación De las Tortugas Marinas.200o pág.

Carr, A.; Carr M. \& A. Meylan (1990). Ecología y Migración de las Tortugas Marinas, 7. Colonia de Tortuga Verde en el Caribe Occidental.

Lagueux, Cynthia (2005). Estrategia de conservación de la Tortuga verde.

Moll, D. (1986; et al., 1996).Valoración económica de las tortugas marinas. 697p.

Nietschman, B. (1995). The cultural context off Sea Turtle subsistence hunting in the Caribbean and problems caused by commercial exploration. In: Biology and Conservation off Sea Turtles. Edited by Karen A. Bjorndal. Washington. 439-435 p. 